Article

\title{
Wave Patterns of Gravity-Capillary Waves from Moving Localized Sources
}

\author{
Vladimir Gnevyshev $1,+, \ddagger(\mathbb{D})$ and Sergei Badulin $1,2, *, \ddagger \mathbb{D}$ \\ 1 Shirshov Institute of Oceanology, Russian Academy of Sciences, 117997 Moscow, Russia; \\ avi9783608@gmail.com \\ 2 Skolkovo Institute of Science and Technology, 121205 Moscow, Russia \\ * Correspondence: badulin.si@ocean.ru; Tel.: +7-499-124-7565 \\ + Current address: Nakhimovsky Pr. 36, 117997 Moscow, Russia. \\ $\ddagger$ These authors contributed equally to this work.
}

Received: 2 November 2020; Accepted: 22 November 2020; Published: 24 November 2020

check for updates

\begin{abstract}
We study wave patterns of gravity-capillary waves from moving localized sources within the classic setup of the problem of ship wakes. The focus is on the co-existence of two wave systems with opposite signatures of group velocity relative to the localized source. It leads to the problem of choice of signs for phase functions of the gravity ("slow") and capillary ("fast") branches of the dispersion relation: the question generally ignored when constructing phase patterns of the solutions. We detail characteristic angles of the wake patterns: (i) angle of demarcation of gravity and capillary waves-"the phase Mach" cone, (ii) angle of the minimal group velocity of gravity-capillary waves- "the group Mach" cone, (iii, iv) angles of cusps of isophases that appear after a threshold current speed. The outer cusp cone is naturally associated with the classic cone of Kelvin for pure gravity waves. The inner one results from the effect of capillarity and tends to the "group Mach" pattern at high speeds of current. Amplitudes of the wave patterns are estimated within the recently proposed approach of reference functions for the problem of propagation of packets of linear dispersive waves. The effect of shape is discussed for elliptic reference sources.
\end{abstract}

Keywords: gravity-capillary waves; ship wake; Kelvin angle; reference solutions

\section{Introduction}

The problem of wavefield behind a moving source is well-known in various domains of modern physics and, in particular, in the dynamics of the atmosphere and ocean [1]. The brightest and deepest for understanding rich physics is the example of ship waves, first considered in the works of Lord Kelvin [2-5]. At the turn of the 20th century, Kelvin and his followers created the basis for many works developing the classical results (e.g., [6,7]).

Patterns of phases of linear waves can be constructed in a relatively simple and general way [8]. In what follows, we assume a motionless source and the flow velocity $\mathbf{U}=(U, 0,0)$ directed along the $x$-axis in the positive direction. We restrict ourselves to the case of two-dimensional waves, which include surface water waves, Rossby waves, and internal gravity waves in a depth-limited ocean (atmosphere). Stationarity condition in the reference frame associated with the source

$$
\omega(\mathbf{k})+k U=0
$$

allows one to determine the transverse component of the wave vector $\mathbf{k}=(k, l)$ by specifying the longitudinal component $k$, which is usually associated with the size of the source. The phase $\varphi=\mathbf{k x}$ 
at an arbitrary point $\mathbf{r}=(x, y)$ is determined from the condition of stationarity (1), i.e., for the conditional extremum of the function

$$
\tilde{\varphi}=\mathbf{k} \mathbf{x}-t(\omega(\mathbf{k})+k U)
$$

The Lagrange multiplier $t$ in (2) can be interpreted as the propagation time of a wave packet with a constant group velocity [9], since the conditional extremum of the phase $\varphi$ is realized at

$$
x=t\left(\frac{\partial \omega}{\partial k}+U\right) ; \quad y=t \frac{\partial \omega}{\partial l} .
$$

The isophases $\varphi=$ const provide a visual picture of the distribution of the wave troughs and crests in a stationary wake created by the source. Note that the straightforward implementation of this approach can lead to non-physical results when the wave pattern appears where it should not be. The reason for this may be the arbitrariness of the choice of the sign of the phase $\varphi$. Formally, $\varphi_{+}=\mathbf{k x}$ and $\varphi_{-}=-\mathbf{k x}$ are equal in rights. Usually, they refer to the principle of causality for choosing the sign of the phase and linking it with the positiveness of the time $t$.

The situation becomes somewhat more complicated when the stationarity condition (1) has two (or more) solutions. Lord Rayleigh [10] considered one of such cases for standing gravity-capillary waves on the surface of running water. For the flow speed higher than the minimum wave velocity $C_{m}=\sqrt{2 g / \sigma} \approx 23 \mathrm{~cm} \cdot \mathrm{s}^{-1}$ (phase and group speeds are the same) the Equation (1) has two roots corresponding to "slow" gravitational and "fast" capillary waves. The group velocities of these waves relative to a stationary source have opposite signatures: the energy of capillary waves runs upstream, while the energy of the gravitational waves lags behind. Rayleigh [10] accounted for this feature in the one-dimensional problem while discussed the solution for the two-dimensional case "by analogy".

The group velocity of waves is considered as an essential quantity of energy transfer since works of Hamilton [11]. It does not enter explicitly into the well-known expressions for the classic Kelvin's solution of pure gravity ship waves [3,4]. The Lighthill [8] interpretation of the Kelvin angle operates with a fixed ratio of $1 / 2$, thus emphasizing the physical difference of the phase and group propagation. This fact alone results in the occurrence of the universal stationary wake patterns in the form of arcs of chevron.

The dispersion of pure capillary waves $\left(\omega(\mathbf{k}) \sim|\mathbf{k}|^{3 / 2}\right)$ is positive, i.e., group velocity is larger than the phase one, and the corresponding wave patterns are not so peculiar as the Kelvin wake ones with their two systems of transverse and divergent waves. In a sense, the wave patterns look trivial. This visual simplicity disappears when passing to gravity-capillary waves where the above wave patterns represent asymptotics at very short and very long waves. The "intermediate" (in words of [12]) range of gravity-capillary waves turns out to be rich in non-trivial kinematic and dynamical effects. Two characteristic scales, minima of phase and group velocities, provide the dimensionless quantities of the Mach numbers. The "phase Mach number" $U_{*}=U / C_{m}$ demarcates gravity and capillary branches of the ship wake. At relatively high $U_{*} \gtrsim 1.938$ singularities of the phase patterns of the ship wake arise as cusps resembling the well-known gussets of Kelvin. A new family of cusps emerges as a feedback of the wave speed minimum, that is, as a result of the coupling of gravity and capillarity effects. The "group Mach number" $U / C_{g, \min }\left(C_{g, \min }\right.$ is minimal group velocity of gravity-capillary waves) forms a cone where diverging capillary and gravity waves cannot enter. This Mach cone is observed in laboratory and field experiments, e.g., ([13]).

The reference solution approach $[9,14]$ resolves the problem of assessment of wake amplitudes under quite mild physical assumptions including wave caustics. At loci of the group velocity minima this method predicts the effect of quasi-dispersion [14], i.e., slow decay of wave amplitude with distance from the source. The observability of this effect is questionable under typical scales of wake but still worth to be mentioned in the context of the previously used asymptotic approaches (e.g., $[13,15])$ and the effect of the "group Mach" [13]. 
In contrast to the "point-wise" estimates of wave amplitudes with conventional asymptotic approaches (e.g., the stationary phase method), the reference solution method describes an evolution of a finite perturbation of elliptic shape. Thus, the effect of the shape on the wake patterns can be discussed with explicit formulas in hand.

In Section 2 the mathematical and physical background for the problem of gravity-capillary waves on running water is presented. The corresponding solutions will be formulated in Section 3 for phase patterns and asymptotic dependencies of wave amplitudes. Discussion of Section 4 is devoted to general aspects of the problem of linear wave propagation and their implications in geophysical hydrodynamics.

\section{Materials and Methods}

\subsection{Kinematics of Linear Gravity-Capillary Waves in Running Water}

\subsubsection{Ray Equations and Isophase Patterns}

This part follows the well-known results of ship wave kinematics used by Thomson [4] himself and his followers (e.g., [1,8]). We consider a point-wise source of perturbation of a stationary current with constant speed $U$ directed along the axis $x$ in positive direction. The source generates gravity-capillary waves which frequencies and wavenumbers obey the well-known dispersion relation [16] with the Doppler shift taken into account

$$
\Omega=\sqrt{g|\mathbf{k}|+\delta|\mathbf{k}|^{3}}+k U
$$

Gravity acceleration $g=981 \mathrm{~cm} \cdot \mathrm{s}^{-2}$ and kinematic water surface tension $(\delta=\gamma / \rho \approx$ $74 \mathrm{~cm}^{3} \cdot \mathrm{s}^{-2} \gamma \approx 74 \mathrm{dyn} \cdot \mathrm{cm}^{-1}, \rho=1 \mathrm{~g} \cdot \mathrm{cm}^{-3}$ is water density) provide spatial and temporal scales of the problem

$$
L=\delta^{1 / 2} g^{-1 / 2}=0.275 \mathrm{~cm} ; \quad T=\delta^{1 / 4} g^{-3 / 4}=0.0167 \mathrm{~s}
$$

associated with the wavelength $\lambda_{m}=2 \pi L=1.73 \mathrm{~cm}$ and wave period $T_{m}=\sqrt{2} \pi / T=0.074 \mathrm{~s}$ of minimal wave velocity $C_{m}=\sqrt{2}(g \delta)^{1 / 4}=\sqrt{2} L / T=23.2 \mathrm{~cm} \cdot \mathrm{s}^{-1}$ [17]. The dimensionless wavenumber $\kappa$ and frequency $\sigma$ are defined as

$$
\kappa=L|\mathbf{k}|, \quad \sigma=T \Omega .
$$

The corresponding dispersion relation (4) becomes

$$
\sigma=\sqrt{\kappa+\kappa^{3}}-\sqrt{2} U_{*} \kappa \cos \theta .
$$

with dimensionless velocity (phase Mach number)

$$
U_{*}=\frac{U}{\sqrt{2}} \frac{T}{L}=\frac{U}{C_{m}}
$$

and angle $\theta$ counted clockwise from negative direction of axis $x$. The condition of stationarity (1) can be parameterized by $\theta$ as follows

$$
\kappa_{x}=-\kappa \cos \theta, \quad \kappa_{y}=\kappa \sin \theta, \quad \cos \theta=\frac{\sqrt{\kappa+\kappa^{3}}}{\sqrt{2} \kappa U_{*}} .
$$

Dimensionless coordinates as functions of dimensionless time $\tau$

$$
\xi=x / L ; \quad \eta=y / L ; \quad \tau=t / T
$$


of the stationary phase can be calculated from the ray Equations (3) as $\mathbf{x}=t \nabla_{\mathbf{k}} \Omega$

$$
\begin{array}{ll}
\xi= & \frac{\tau}{2 \sqrt{\kappa+\kappa^{3}}}\left(1+3 \kappa^{2}\right) \cos \theta+\sqrt{2} U_{*} \tau \\
\eta= & \frac{\tau}{2 \sqrt{\kappa+\kappa^{3}}}\left(1+3 \kappa^{2}\right) \sin \theta .
\end{array}
$$

Define the phase function $\varphi_{ \pm}= \pm(k x+l y)= \pm\left(\kappa_{x} \xi+\kappa_{y} \eta\right)$ and write it accounting for the condition of stationarity (1)

$$
\varphi_{ \pm}=\mp \frac{\tau\left(\kappa-\kappa^{3}\right)}{2 \sqrt{\kappa+\kappa^{3}}}
$$

Trajectories (11) and (12) can be written as functions of phase $\varphi_{ \pm}$

$$
\begin{aligned}
& \xi=\mp \frac{\varphi \cos \theta}{\kappa-\kappa^{3}}\left(1+3 \kappa^{2}-4 \kappa U_{*}^{2}\right) \\
& \eta=\mp \frac{\varphi \sin \theta}{\kappa-\kappa^{3}}\left(1+3 \kappa^{2}\right) .
\end{aligned}
$$

Expressing wavenumber $\kappa$ in terms of angle $\theta$ one gets from (9) two roots

$$
\kappa_{1,2}=U_{*}^{2} \cos ^{2} \theta \pm \sqrt{U_{*}^{4} \cos ^{4} \theta-1}
$$

Each root requires special choice of signature of the phase function $\varphi$ (13). This step is usually associated with the principle of casuality. An equivalent formulation can be proposed based on the group velocity signatures corresponding to the roots $\kappa_{1,2}$. Note that the critical value $\kappa_{m}=1$ determines minimal value of phase velocity, i.e., merging of the roots (16) along direction $\theta$ in wavevector space. For the gravity branch $\left(\kappa_{1}<1\right)$ the group velocity is directed downstream that dictates the choice of sign ' - ' in $\varphi$ (13). Alternatively, the sign ' + ' appears for the capillary branch $\left(\kappa_{2}>1\right)$.

The line $\left|U_{*} \cos \theta\right|=1$ demarcates two families of isophases in wavevector space. In the coordinate space $(\xi, \eta)$ the merging of gravity and capillary branches occurs at the asymptotes of isophases $\varphi_{1,2}=$ const $_{1,2}[1]$

$$
\tan \beta_{m}=\left(\frac{\eta}{\xi}\right)_{m}=\mp \frac{\tan \theta_{m}}{U_{*}^{2}-1}= \pm \sqrt{U_{*}^{2}-1} \quad \text { or } \quad \sin \beta_{m}=-\cos \theta_{m}=\frac{1}{U_{*}} .
$$

2.1.2. Minimal Group Velocity and Cusps of Isophases $\varphi_{ \pm}=$const

One more characteristic scale of the wake patterns can be related to the minimum of group velocity of the gravity-capillary waves. For water waves it gives

$$
C_{g, \text { min }}=\min \left(C_{g}\right)=\frac{1}{2} \frac{\sqrt{3}-1}{(2 / \sqrt{3}-1)^{1 / 4}} C_{m} \approx 0.768 C_{m} \approx 17.8 \mathrm{~cm} \cdot \mathrm{s}^{-1} .
$$

for dimensionless wavenumber $\kappa_{\min }=\sqrt{2 / \sqrt{3}-1}$. The corresponding phase velocity and, hence, the current speed $C_{p, \min }=(2 \sqrt{3}-3)^{-1 / 4} C_{m} \approx 28.1 \mathrm{~cm} \cdot \mathrm{s}^{-1}$ is just $20 \%$ higher than the minimal $C_{m}$. The minimum of the group velocity is closely related to the condition of singularity of parametric curves of isophases (14) and (15)

$$
\frac{d \xi}{d \theta}=0 ; \quad \frac{d \eta}{d \theta}=0
$$


It leads to the polynomial equation in our dimensionless variables (cf. [15] Equation (20)) or ([12] Equation (16))

$$
Z_{6}\left(\kappa, U_{*}\right)=3 \kappa^{6}-12 \kappa^{5} U_{*}^{2}+23 \kappa^{4}-24 \kappa^{3} U_{*}^{2}+9 \kappa^{2}+4 \kappa U_{*}^{2}-3=0
$$

that can be re-written in quite transparent form as a dependency of dimensionless speed $U_{*}$ on wavenumber in the cusp

$$
U_{*(\text { cusps })}^{2}=\frac{1}{4 \kappa} \frac{3 \kappa^{6}+23 \kappa^{4}+9 \kappa^{2}-3}{3 \kappa^{4}+6 \kappa^{2}-1}
$$

Dependencies $Z_{6}$ and $U_{*(\text { cusps })}$ are shown in Figure 1a,b. The cusps appear when $U_{*}$ exceeds a threshold value $U_{*} \approx 1.938$ at the corresponding dimensionless wavenumber $\kappa_{t h r} \approx 0.276$ (water wave wavelength $\lambda_{\text {cusp }} \approx 6.27 \mathrm{~cm}$ ) that is a root of the cubic equation

$$
3 \kappa^{6}+\kappa^{4}+13 \kappa^{2}-1=0 .
$$

For a given $U_{*}$, two solutions for $\kappa_{\text {cusp }}$ exist. The longer wave solution correspond to the cusp of the classic Kelvin pattern for deep water gravity waves where two system of transversal and diverging waves merge. The shorter wave solution is limited by the condition of vanishing the denominator in (21). It is easy to show that this is exactly the condition of the group velocity minimum $\partial^{2} \sigma / \partial \kappa^{2}=0$ $\left(\partial^{2} \Omega / \partial|\mathbf{k}|^{2}=0\right)$, i.e., $\kappa_{\min }=\sqrt{2 / \sqrt{3}-1} \approx 0.393$. Below we refer to this family of cusps as capillary one as far as it results from the capillarity effect. Plugging (21) into ray Equations (14) and (15) one gets directions of wavevectors at the cusp points ( $\kappa_{\mathcal{C}}$ is the wavenumber at the cusp point)

$$
\tan ^{2} \theta_{\text {cusp }}=\frac{\left(\kappa_{c}^{2}-1\right)^{2}\left(1+3 \kappa_{c}^{2}\right)}{2\left(1+\kappa_{c}^{2}\right)\left(3 \kappa_{c}^{4}+6 \kappa_{c}^{2}-1\right)}
$$

and the direction of the cusp line in the coordinate space

$$
\tan ^{2} \beta_{\text {cusp }}=\left(\frac{1+3 \kappa_{c}^{2}}{1+3 \kappa_{c}^{2}-4 \kappa U_{*}^{2}}\right)^{2} \tan ^{2} \theta_{\text {cusp }}=\frac{\left(1+3 \kappa_{c}^{2}\right)^{3}\left(3 \kappa_{c}^{4}+6 \kappa_{c}^{2}-1\right)}{2\left(1+\kappa_{c}^{2}\right)^{3}\left(6 \kappa_{c}^{2}-2\right)^{2}}
$$

Dependencies of angles $\theta_{\text {cusp }}, \beta_{\text {cusp }}$ are shown in Figure 1c. The upper branch of the blue curve and lower one of the red correspond to the well-known cusps of gravity waves that conform the classic Kelvin pattern. In the limit $U_{*} \rightarrow \infty$ the cusps are located at the straight lines with the Kelvin angle $\beta_{\text {Kelvin }}=19.47^{\circ}$. The wavevectors at the cusps are tending to the universal angle $\theta_{\text {Kelvin }}=35.26^{\circ}$.

The remaining branches in Figure $1 \mathrm{c}$ are tending to "trivial" values $\beta_{\text {cusp }} \rightarrow 0$ and $\theta_{\text {cusp }} \rightarrow 90^{\circ}$. The dependency of the inner (capillary) cusp cone angle $\beta_{\text {cusp }}$ on the current speed is an intriguing issue. In the limit of high speeds $U_{*} \rightarrow \infty$ it is tending to the inverse Mach number constructed of the current speed and the minimal group velocity as shown in Figure 1d

$$
\tan \beta_{\text {group }}=\frac{0.7681}{U_{*}} .
$$

As shown below, the direction of minimal group velocity $C_{g, \min }(18)$ is one of quasi-dispersion (51) with peculiar dependence of wave amplitudes on distance [9]. 


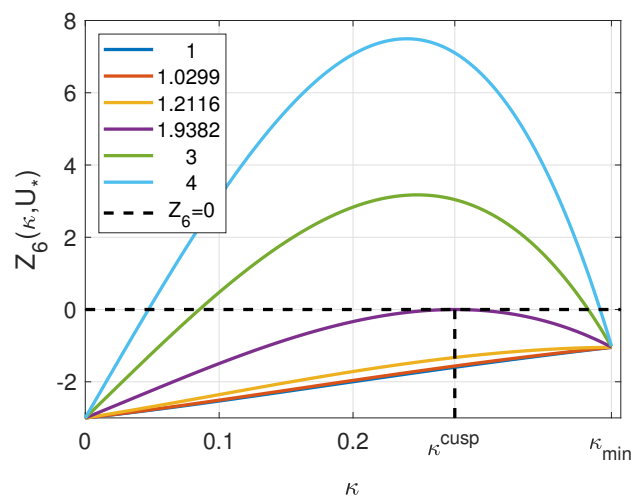

(a)

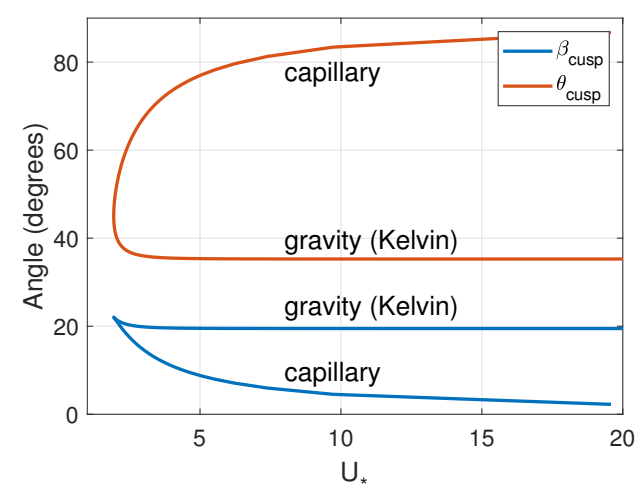

(c)

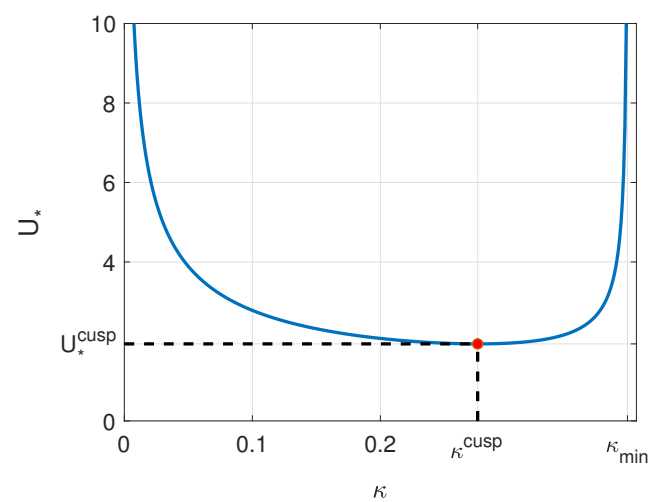

(b)

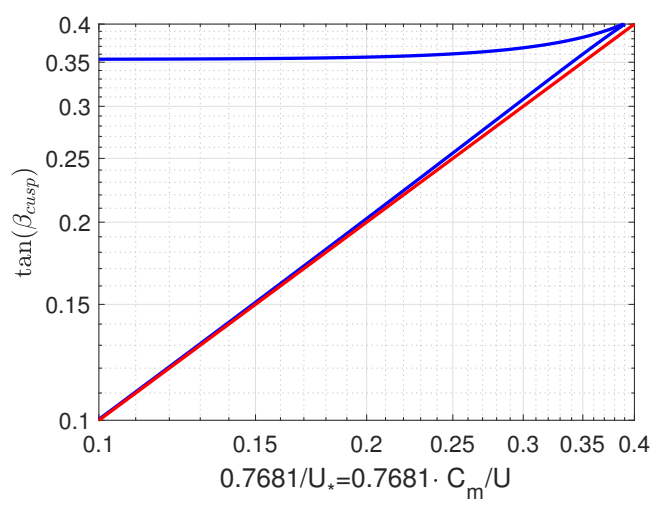

(d)

Figure 1. (a) Polynomial $Z_{6}\left(\kappa, U_{*}\right)(20)$ at different values of $U_{*}$ (see legend); (b) Dependence of $U_{*}$ on wavenumber of cusps (21); (c) Angles of cusp lines in coordinate (blue) and wave vector spaces (red); (d) Dependence of the cusp line tangent on the inverse Mach number $0.7681 C_{m} / U_{*}$ (blue line), the diagonal is shown in red.

Finally, we have three characteristic angles of the gravity-capillary wake patterns:

1. Angle $\beta_{m}$ (17) demarcates families of gravity and capillary waves at low speeds $U_{*} \lesssim 1.938$. After the appearance of cusps of isophases, these two families can overlap as shown below. This angle can be naturally related to the Mach number constructed of the minimal phase velocity $C_{m}$;

2. Cusps of isophases occur at relatively high speeds $U_{*} \gtrsim 1$ 1.938. In addition to the classic Kelvin pattern where diverging and transversal systems merge a new family of cusps, fan waves, in the words of [15], conform a divergent-fan pattern. Loci of the cusp is a cone with one of the angle $\beta_{\text {cusp }}$ (Equation (24), when Equation (20) has two solutions), while the fan wave fronts aligned along the demarcation line (angle $\beta_{m}$ ). Asymptotically, this cone tends to the Mach one constructed of the minimal group velocity of the gravity-capillary waves $C_{g, \text { min }}$. It should be stressed that this cone is due to the capillarity effect in the domain of gravity waves;

3. At the limit $U_{*} \rightarrow \infty$ the cone where transversal and divergent gravity waves are merging is tending to its classical Kelvin value $\beta_{K}=\arcsin (1 / 3) \approx 19.47^{\circ}$. Thus, there is a continuous transition of this pattern between pure gravity and gravity-capillary waves.

\subsection{Wave Amplitudes in the Gravity-Capillary Ship Wake}

\subsubsection{The Reference Solutions Approach}

Estimates of wave amplitudes in gravity-capillary wake follow the previous authors' papers on the so-called approach of reference solutions $[9,14]$. Here, we fix main points of the method. 
The idea of the method has been exploited many times since Kelvin [2]. The initial condition for a wave variable $f$ (below we assume $f$ to be free surface elevation) is set in the form

$$
f(\mathbf{x})=\operatorname{Re}\left[F(\mathbf{x}) \exp \left(\mathbf{i k}_{0} \mathbf{x}\right)\right]
$$

i.e., as a quasi-monochromatic wave packet with a carrier wavevector $\mathbf{k}_{0}$ and "slow" modulation function $F(\mathbf{x})$. Then, a special "reference function" $F(\mathbf{x})$ is chosen for effective manipulations with linear solutions (e.g., [18], for one-dimensional case). The gaussian shape of $F$ allows one for treating (26) as a superposition of linear harmonics with the gaussian distribution of amplitudes. The temporal evolution of this distribution is governed by the effect of dispersion of harmonics, that is, by properties of particular dispersion relationship (in our case, by Equation (4)). The distribution is assumed to be narrow in wavevector space and the dispersion effect is accounted up to the quadratic term in the formally small bandwidth parameter. The quadratic form appears as an argument of the corresponding exponent for an arbitrary time. It allows one for presenting wave packet evolution both in coordinate and wavevector spaces as one of the gaussian shape with parameters of the quadratic form being dependent on time.

The approximate solution can be written as follows [14]

$$
f(\mathbf{x}, t)=\frac{1}{D^{1 / 2}\left(\mathbf{k}_{0}, t\right)} \exp \left(-\frac{\Lambda\left(\mathbf{k}_{0}, \zeta, t\right)}{2 D^{2}\left(\mathbf{k}_{0}, t\right)}\right) \times \cos \left[\vartheta_{0}\left(\mathbf{k}_{0}, \mathbf{x}, t\right)+\vartheta_{1}\left(\mathbf{k}_{0}, \zeta, t\right)+\vartheta_{2}\left(\mathbf{k}_{0}, \zeta, t\right)\right]
$$

where "fast" $\mathbf{x}$ and "slow" $\zeta$ coordinates are used to discriminate "fast" effect of wave propagation in $\mathbf{x}$-space and a "slow" dispersion of the wave packet of a finite bandwidth relatively to the carrier harmonics $\mathbf{k}_{0}$.

The "fast" amplitude factor

$$
D^{2} \equiv|\operatorname{det}(\mathbf{B})|^{2}=\left\{\left[1+t^{2}\left(\mu_{x y}^{2}-\mu_{x} \mu_{y}\right)\right]^{2}+t^{2}\left(\mu_{x}+\mu_{y}\right)^{2}\right\}
$$

describes the overall changes of wave packet amplitude due to its finite bandwidth and can be seen as a multi-dimensional generalization of the corresponding amplitude factor in the method of stationary phase [14]. The second differentials

$$
\mu_{x}=\left.\Omega_{k k}^{\prime \prime}\right|_{\mathbf{k}=\mathbf{k}_{0}}(\Delta k)^{2} ; \quad \mu_{y}=\left.\Omega_{l l}^{\prime \prime}\right|_{\mathbf{k}=\mathbf{k}_{0}}(\Delta l)^{2} ; \quad \mu_{x y}=\left.\Omega_{k l}^{\prime \prime}\right|_{\mathbf{k}=\mathbf{k}_{0}}(\Delta k \Delta l) .
$$

appear in the denominator of (27) quite similarly to the stationary phase method. The function $D$ in the argument of exponential function (27) provides an additional wave packet blurring.

The "slow" coordinate

$$
\zeta=\left(\Delta k\left(x-\left.\Omega_{k}^{\prime}\right|_{\mathbf{k}=\mathbf{k}_{0}} t\right), \Delta l\left(y-\left.\Omega_{l}^{\prime}\right|_{\mathbf{k}=\mathbf{k}_{0}} t\right)\right.
$$

describes deviations of harmonics relative to the carrier wave harmonic $\mathbf{k}_{0}$ that is absent in the stationary phase method.

The quadratic form $\Lambda\left(\mathbf{k}_{0}, \zeta, t\right)$ of exponential function in (27)

$$
\Lambda\left(\mathbf{k}_{0}, \zeta, t\right)=\zeta_{1}^{2}\left[1+t^{2}\left(\mu_{x y}^{2}+\mu_{y}^{2}\right)\right]+\zeta_{2}^{2}\left[1+t^{2}\left(\mu_{x y}^{2}+\mu_{x}^{2}\right)\right]-2 \zeta_{1} \zeta_{2} t^{2} \mu_{x y}\left(\mu_{x}+\mu_{y}\right)
$$

preserves gaussian shape of the wave train modulation. Its isolines are ellipses, the parameters of which (orientation and eccentricity) vary with time.

In addition to the carrier wave phase $\vartheta_{0}$

$$
\vartheta_{0}=\mathbf{k}_{0} \mathbf{x}-\Omega_{0} t
$$


two phase shifts $\vartheta_{1}, \vartheta_{2}$ appear in (27). The first one $\vartheta_{1}$ shifts phases of all the wavetrain components by the same value

$$
\vartheta_{1}\left(\mathbf{k}_{0}, t\right)=-\frac{1}{2} \arctan \left[\frac{t\left(\mu_{x}+\mu_{y}\right)}{1+t^{2}\left(\mu_{x y}^{2}-\mu_{x} \mu_{y}\right)}\right] .
$$

For omnidirectional propagation and in special case of quasi-dispersion $\left(\mu_{x y}^{2}-\mu_{x} \mu_{y}=0\right.$, see [14]) $\vartheta_{1}$ tends to the well-known result of the stationary phase method $\vartheta_{1}= \pm \pi / 4$ (e.g., [19]).

Phase shift $\vartheta_{2}\left(\mathbf{k}_{0}, \zeta, t\right)$ depends on "slow" coordinate $\zeta$

$$
\begin{aligned}
\vartheta_{2}\left(\zeta, \mathbf{k}_{0}, t\right) & =\frac{1}{2 D^{2}}\left\{\zeta_{1}^{2}\left[t^{3} \mu_{y}\left(\mu_{x y}^{2}-\mu_{x} \mu_{y}\right)-t \mu_{x}\right]+\zeta_{2}^{2}\left[t^{3} \mu_{x}\left(\mu_{x y}^{2}-\mu_{x} \mu_{y}\right)-t \mu_{y}\right]\right. \\
& \left.-2 \zeta_{1} \zeta_{2} t \mu_{x y}\left[1+t^{2}\left(\mu_{x y}^{2}-\mu_{x} \mu_{y}\right)\right]\right\} .
\end{aligned}
$$

It vanishes for the wavetrain carrier harmonic $(\zeta=0)$ and changes peripheral harmonics when $\zeta \neq 0$. For deep water waves $\vartheta_{2}(\zeta, \mathbf{k}, t)$ is decaying as $t^{-1}$.

One can restrict our consideration by the approximation of function $f$ in (27)

$$
f(\mathbf{x}, t) \approx \frac{1}{D^{1 / 2}\left(\mathbf{k}_{0}, t\right)} \exp \left(-\frac{\Lambda\left(\mathbf{k}_{0}, \zeta, t\right)}{2 D^{2}\left(\mathbf{k}_{0}, t\right)}\right) \cos \left[\vartheta_{0}\left(\mathbf{k}_{0}, \mathbf{x}, t\right)\right] .
$$

In terms of dimensionless variables, one has for parameters of dispersion

$$
\begin{aligned}
t^{2}\left(\mu_{x y}^{2}-\mu_{x} \mu_{y}\right) & =-\tau^{2} \frac{\Delta_{x}^{2} \Delta_{y}^{2}}{8} \frac{\left(6 \kappa^{2}+3 \kappa^{4}-1\right)\left(1+3 \kappa^{2}\right)}{\left(\kappa+\kappa^{3}\right)^{2} \kappa} \\
t \mu_{x} & =\tau \frac{\Delta_{x}^{2}}{4}\left[\frac{\left(3 \kappa^{4}+6 \kappa^{2}-1\right) \cos ^{2} \theta+\left(6 \kappa^{4}+8 \kappa^{2}+2\right) \sin ^{2} \theta}{\left(\kappa+\kappa^{3}\right)^{3 / 2}}\right] \\
t \mu_{y} & =\tau \frac{\Delta_{y}^{2}}{4}\left[\frac{\left(3 \kappa^{4}+6 \kappa^{2}-1\right) \sin ^{2} \theta+\left(6 \kappa^{4}+8 \kappa^{2}+2\right) \cos ^{2} \theta}{\left(\kappa+\kappa^{3}\right)^{3 / 2}}\right] .
\end{aligned}
$$

Note that dimensionless bandwidth components in (36)-(38) are scaled by the the capillarity length $L$ (5)

$$
\Delta_{x}=L \Delta k ; \quad \Delta_{y}=L \Delta l .
$$

This is in contrast with the problem of pure gravity ship waves where the scaling is arbitrary and, generally, refers to the carrier wavelength.

The determinant $D$ can be presented in the form

$$
D^{2}=(1-A)^{2}+B^{2}
$$

where

$$
\begin{aligned}
A & =\frac{\varphi^{2} \Delta_{x}^{2} \Delta_{y}^{2}}{2} \frac{\left(3 \kappa^{4}+6 \kappa^{2}-1\right)\left(1+3 \kappa^{2}\right)}{\left(\kappa^{2}-1\right)^{2}\left(1+\kappa^{2}\right)^{2} \kappa^{4}} \\
B^{2} & =\frac{\varphi^{2}}{\kappa^{2}\left(\kappa^{2}-1\right)^{2}}\left[\Delta_{x}^{2}\left(C \cos ^{2} \theta+K \sin ^{2} \theta\right)+\Delta_{y}^{2}\left(C \sin ^{2} \theta+K \cos ^{2} \theta\right)\right]^{2}
\end{aligned}
$$

and

$$
C=\frac{3 \kappa^{4}+6 \kappa^{2}-1}{2\left(1+\kappa^{2}\right)} ; \quad K=1+3 \kappa^{2}
$$

With the stationarity condition (9) wave amplitude (determinant $D$ ) can be presented as function of angle $\theta$ (or angle $\beta=\arctan (y / x)$ ) and dimensionless $U_{*}$, the "phase Mach" number. The effect of such a transformation is different for gravity and capillary family of the wake. This justifies our 
appeal to the limiting cases of the dispersion relation (4), i.e., to the cases of pure gravity and pure capillary waves.

\subsubsection{Limiting Cases of Wave Patterns. The Effect of Wave Dispersion on Wave Patterns}

The classic case of pure gravity waves has been studied in detail since Lord Kelvin's works [2-5], while the alternative case of pure capillarity did not attract too much attention so far. This seems to be a serious gap since the change of signature of the wave dispersion leads to fundamental differences in the geometry of the patterns and the distribution of wave amplitudes. Here, we outline very few features of the issue leaving the extended discussion for future publications.

The limiting cases can be easily derived from general dimensionless relationships (9)-(16). Scaling parameters for pure gravity and capillary waves can be chosen arbitrarily for the power-like dispersion relations where specific temporal or spatial scales are absent. Thus, one can leave $C_{m}$ and, correspondingly, $U_{*}(9)-(16)$ or propose an alternative scaling for a particular problem to be solved. Both solutions are equally acceptable for the analysis of wave kinematics.

Stationarity condition (9) provides a pair of roots $\kappa_{1,2}$ obeying $\kappa_{1} \kappa_{2}=1$. Thus, the formally small or large roots at $U_{*} \rightarrow \infty$ describe alternative limiting cases of pure gravity $\left(\kappa_{1} \rightarrow\left(2 U_{*} \cos \theta\right)^{-2} \rightarrow 0\right)$ and capillary waves $\left(\kappa_{2} \rightarrow\left(2 U_{*} \cos \theta\right)^{2} \rightarrow \infty\right)$. Plugging these asymptotic expressions into trajectory Equations (14) and (15), one can easily get the well-known parametric equations for isophases for pure gravity waves (e.g., [9], Equation (26))

$$
\begin{array}{lc}
\xi= & 2 U_{*}^{2} \varphi \cos \theta\left(2-\cos ^{2} \theta\right) \\
\eta= & 2 U_{*}^{2} \varphi \sin \theta \cos ^{2} \theta
\end{array}
$$

and their pure capillary counterparts within the generic scaling of this paper (5)-(8)

$$
\begin{array}{lc}
\xi= & \frac{\varphi}{2 U_{*}^{2} \cos ^{3} \theta}\left(2-3 \cos ^{2} \theta\right) \\
\eta= & \frac{3 \varphi \sin \theta}{2 U_{*}^{2} \cos ^{2} \theta} .
\end{array}
$$

One should emphasize a sort of similarity of pure gravity and capillary patterns: both the well-known Kelvin gusset of gravity waves and the parabolic pattern of capillary waves in Figure 2 are independent of the dimensionless current speed $U_{*}$. The speed magnitude just re-scales these patterns in both directions but does not affect their geometry. This trivial conclusion results from the above mentioned fact of disappearance of characteristic scale (e.g., $C_{m}$ ) when passing to limiting cases of long (gravity) or short (capillary) waves.

The qualitative dissimilarity of the patterns in Figure 2 results, evidently, from different signatures of wave dispersion: negative for gravity and positive for capillary waves. For power-law dispersion in unmoving media $v=\alpha \kappa^{n}$ cf. (7) with a constant $\alpha$ and positive $n$ the ratio of group and phase velocity

$$
r=\frac{\partial v}{\kappa} \frac{\kappa}{v}
$$

is constant. At $r<1$ (negative dispersion, case of gravity waves when energy propagates slower than phase) the Lighthill [8] illustration for the Kelvin cone becomes valid. For an arbitrary $r<1$, the downstream cavity in the form of a cone with the corresponding opening angle is filled by waves. In the alternative case of positive dispersion (capillary waves and waves with dispersion Equation (48) and $n>1$ ) when energy propagates upstream the waves do not enter a downstream cavity of parabolic shape (Figure 2b). 

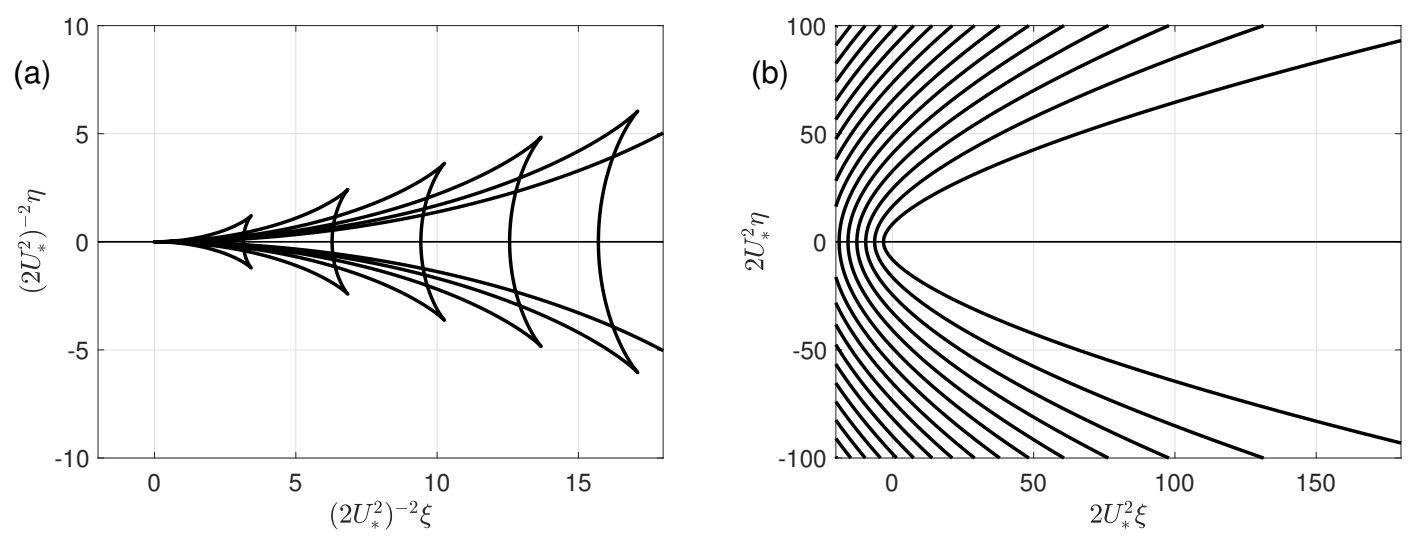

Figure 2. Isophases of pure gravity (a) and pure capillary waves (b) in dimensionless representation (44)-(47).

Dissimilarity in behavior of wave amplitudes requires a special discussion. Here, we just note that an additional physical scale, dimension of the source, in expressions (40)-(43) introduces an additional dimensionless parameter. The Froude number determined by the size of the source $R_{x}$ can be defined in the well-known way (e.g., [8]) for pure gravity waves

$$
F r_{g}^{2}=\frac{U^{2}}{g R_{x}}
$$

Pure capillary waves require a special definition, say, as follows

$$
F r_{c}^{2}=\frac{U^{2} R_{x}}{\delta}
$$

Irrespectively of the definitions (49)-(50), the amplitude dependencies on the current speed (40)-(42) are qualitatively different in the limiting cases: wake amplitude is decaying with speed for pure gravity while it is growing for the capillary counterparts. This delicate issue requires special study. The main focus of the paper is an intermediate range of gravity-capillary waves where both effects of gravity and capillarity compete on equal terms.

\subsubsection{Anomalous Dispersion of Gravity-Capillary Ship Wake}

Explicit Formulas (40)-(43) turn us back to the effect of minimum of group velocity $C_{g, m}$. In the general case, $A \neq 0$ determines the amplitude decay $f \sim \varphi^{-1}$ or $\tau^{-1}$ that is consistent with the result for pure gravity waves (e.g., [9]). The capillarity changes this result qualitatively when $A$ vanish at

$$
\kappa_{\min }^{2}=\frac{2}{\sqrt{3}}-1
$$

that is exactly at the wavenumber of the minimum of group velocity (cf. Equation (18)). It is interesting that the combination of the second derivatives responsible for dispersion of two-dimensional wave packet vanishes as well, i.e., (see Equation (36))

$$
\mu_{x y}^{2}-\mu_{x} \mu_{y}=0
$$

The case of quasi-dispersion has been first described in Gnevyshev and Badulin [14] for Rossby waves in the ocean with homogeneous vertical stratification (buoyancy frequency is constant). The vanishing of the second derivative $\Omega_{k k}^{\prime \prime}$ is just a special case when the omni-directional method of 
stationary phase predicts a slower dispersion of wave packets. Using a more general approach of the reference functions, one has an anomalously slow dispersion $\tau^{-1 / 2}$ in the two-dimensional problem.

It is easy to see that the anomalous (quasi-) dispersion occurs for the direction $\beta_{\text {group }}$ specified in the previous section (25) and for relatively high speeds $U_{*}>(2 \sqrt{3}-3)^{-1 / 4} \approx 1.212$. One should note that amplitudes are vanishing at the demarcation asymptote at $\beta_{m}$ where $\kappa=1$ (17). At the line of quasi-dispersion $\beta_{\text {group }}$ (25) and (52) the amplitudes are not zero but asymptotically decaying slower than in close directions.

\section{Results}

In this section, we present illustrations of the above theoretical analysis for different parameters. The dimensionless speed $U_{*}$, the Mach number related to the minimum of phase speed, determines characteristic wave patterns. Dimensionless coordinates $\xi, \eta$ make distributions of wave phases and amplitudes universal, i.e., independent of the nature of fluids. The dimensionless variable wavenumber $\kappa=L|\mathbf{k}|$ can be treated as the inverse Bond number with wavelength $\lambda$ as a characteristic scale. In our discussion of results, we mostly focus on an intermediate range of waves where both gravity and capillarity effects are equally important. For illustrations of wave phase patterns, we choose characteristic values of the current speed:

1. The phase speed of the first Wilton resonance [20];

2. The phase speed of the minimum group velocity;

3. The threshold value $U_{\text {cusps }}=1.938$ of the isophase cusps;

4. $\quad U_{*}=4$ as a case when families of gravity and capillary branches essentially overlap.

When discussing wave amplitude distributions, we consider just three cases of the above list, avoiding a special case of wave caustics and superposition of different wave systems (gravity and capillary).

\subsection{Phase Patterns at Different Speeds of Current $U_{*}$}

3.1.1. The First Wilton Resonance $U_{*}=\sqrt{3 / 2 \sqrt{2}} \approx 1.03$

A stationary wave pattern appears when the current speed exceeds the minimal phase speed of waves, i.e., for $U_{*}=U / C_{m}>1$. One can start illustrations with the case of the first Wilton resonance [20]. Wavelengths of the Wilton harmonics for water waves $\lambda_{\text {Wilton } 1}=2.44 \mathrm{~cm}$ and $\lambda_{\text {Wilton } 2}=1.22 \mathrm{~cm}$ correspond to rather small excess of the current speed over the minimal speed $C_{m} \approx 23 \mathrm{~cm} \cdot \mathrm{s}^{-1}$, i.e., $U_{*}=\sqrt{3 / 2 \sqrt{2}} \approx 1.03$.

Figure 3a shows two systems of waves. Below, we refer to waves running upstream and shown by red isolines as a capillary family. Their downstream counterparts of gravity family are given in blue. The two families are independent at this current speed, that is, their domains are not overlapping. The demarcation line of the families, the asymptote (17), is shown in magenta. The classic Kelvin cone is given by a black solid line for reference.
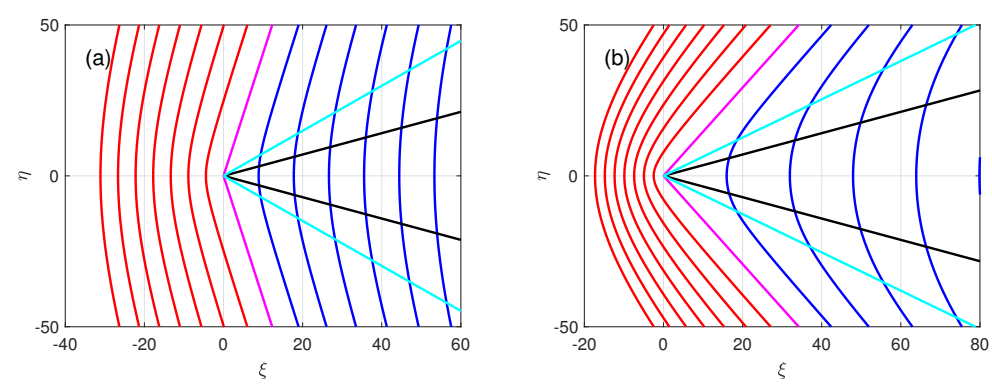

Figure 3. Cont. 

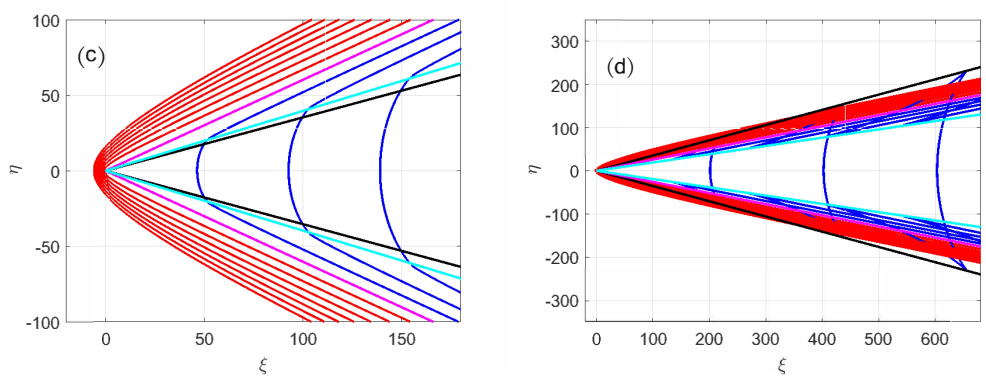

Figure 3. Patterns of the wake isophases (13). (a) $U_{*}=\sqrt{3 /(2 \sqrt{2})} \approx 1.03$, the first Wilton resonance; (b) $U_{*}=(2 \sqrt{3}-3)^{-1 / 4} \approx 1.212$, the minimum of group velocity; (c) $U_{*}=1.938$, appearance of cusps of isophases of gravity family; (d) $U_{*}=4$, merging two families of isophases and appearance of the inner Mach cone. Magenta solid line—the "phase Mach" cone (17), cyan solid line—the "group Mach" cone (25), black line is the classic Kelvin cone.

The cone of "group Mach" $U / C_{g, \min }$ is shown in cyan. It is wider than the Kelvin cone (black lines).

3.1.2. The Minimum of the Group Velocity $U_{*}=(2 \sqrt{3}-3)^{-1 / 4}$

The case of minimal group velocity shows nothing new in the phase distributions. This is the threshold value of $U_{*}$ when the inner Mach cone emerges and the condition of anomalous wave dispersion (52) starts to be satisfied. The "group Mach" cone is still essentially larger than the Kelvin one.

\subsubsection{Cusps of Isophases at $U_{*(\text { cusps })} \approx 1.938$}

As mentioned above, the case of the appearance of singularities of isophases is of special interest. All the characteristic cones (demarcation—magenta, "group Mach" and Kelvin's one are well separated.

\subsubsection{Merging Wave Systems. Inner Mach's cone}

The domains of gravity and capillary families continue to be separated by the demarcation lines up to $U_{*} \approx 3$ when the fan waves match the classic Kelvin cone. At slightly lower $U_{*}$, the domains of capillary and gravity families start to overlap. While the capillary family isophases remain smooth, the gravity family curves form two types of cusps that correspond to two roots of (20) and (21). The longer wave cusp can be naturally related to the well-known caustics of the Kelvin wake of pure gravity waves. The shorter wave cusps (fan waves in words of [15]) are genetically related to the capillarity effect as it has been noted by Moissy and Rabaud [13]. Asymptotically, when $U_{*} \rightarrow \infty$ this Mach cone is shrinking as (24). The dimensionless wavenumbers at the cone are limited by maximal value $\kappa_{\min }=\sqrt{2 / \sqrt{3}-1} \approx 0.393$ when the denominator of (21) vanishes. This wavenumber corresponds to the minimum of the group velocity of gravity-capillary waves as noted above. Thus, the inner cone of the anomalous dispersion (25) and (52) always remains inside the cone of capillary cusps.

\subsection{Wake Amplitudes}

The reference solution method provides analytical solutions (40)-(42) that can be easily converted to graphics. Presenting these figures, the authors are trying to accompany them by comments on possible physical effects governing the amplitude distributions.

The key amplitude factor $D$ depends on two variables: the wavenumber $\kappa$ that is determined by wave direction $\theta$ at a given speed $U_{*}$ (16) and the distance along the ray expressed by time $\tau$ or phase $\varphi$. The first remark to be made is the presence of two terms $A$ and $B$ in the expression of $D(40)$ : the first term on the right-hand side is proportional to phase $\varphi$ in power 4 while the second one is 
just squared phase. Thus, vanishing of $A$ qualitatively changes asymptotical behavior along wave rays (with distance from the source), while vanishing the term $(1-A)$ can lead to an extremum of amplitudes both as functions of distance and angle, and rather peculiar distributions of amplitudes in the ship wake.

The second remark concerns the demarcation line $\kappa=1$ where amplitudes of wake vanish as it is seen from (40)-(42): $A, B$ are singular in this case. The observability of this effect is an interesting point for experimentalists.

In this paper, we present distributions of wave amplitudes for relatively low speeds when these amplitudes are governed by the only wave system at a given coordinate point that is for $U_{*} \leq 1.938$. At higher speeds after singularity occurrence, two (or even three) wave systems can be easily superimposed as it has been done for pure gravity waves in [9]. Here, we restrict ourselves by the simplest case. Note that in this case there is no similarity with the case of pure gravity waves where two different overlapping wave systems (divergent and transversal) always co-exist irrespectively to the current speed value.

\subsubsection{Wakes of Circular Sources}

For the circular shape of the source when $\Delta_{x}=\Delta_{y}$, wave amplitudes (terms $A, B, D$ ) do not depend on angle $\theta$ explicitly. While wavenumber $\kappa$ depends on $\theta$ monotonically basic features of distributions can be investigated qualitatively.

Figure 4 shows the amplitude factor $D^{-1 / 2}\left(\mathbf{k}_{0}, t\right)$ in (35) as function of dimensionless coordinates $(\xi, \eta)$, that is, a relative decrease of particular wave harmonics with the distance along a ray. Wave patterns are shown in two columns: the left panels are zoomed images of the right ones. Colorbars are scaled by the exponent $n$ of $2^{-n}$.

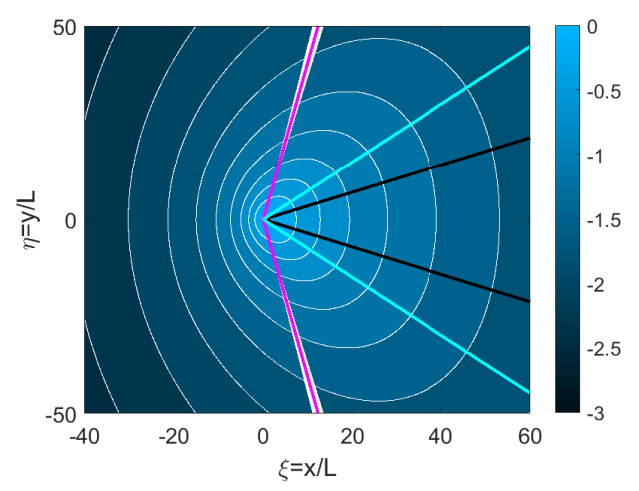

(a)

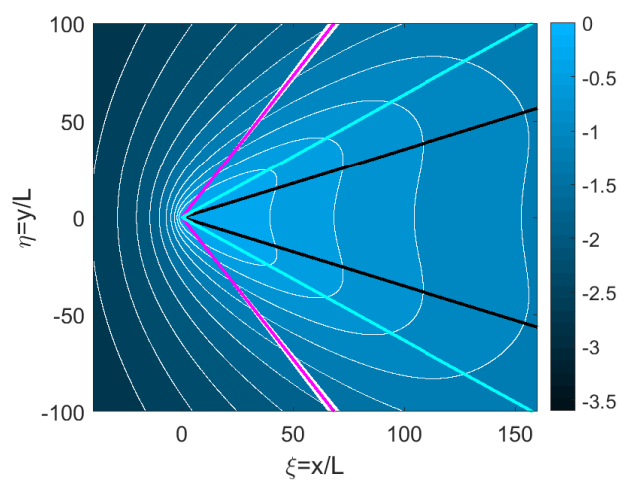

(c)

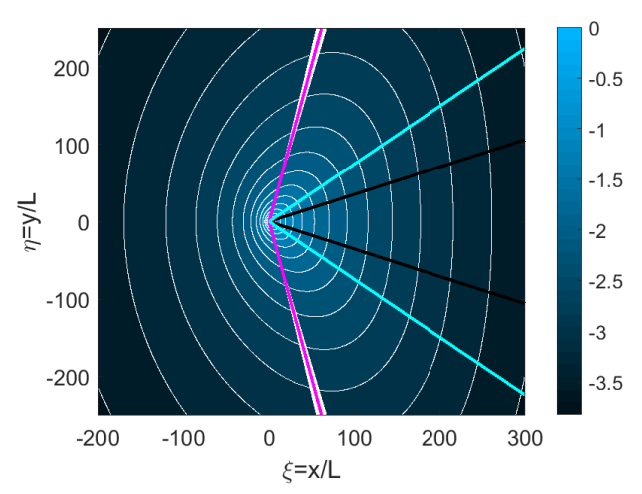

(b)

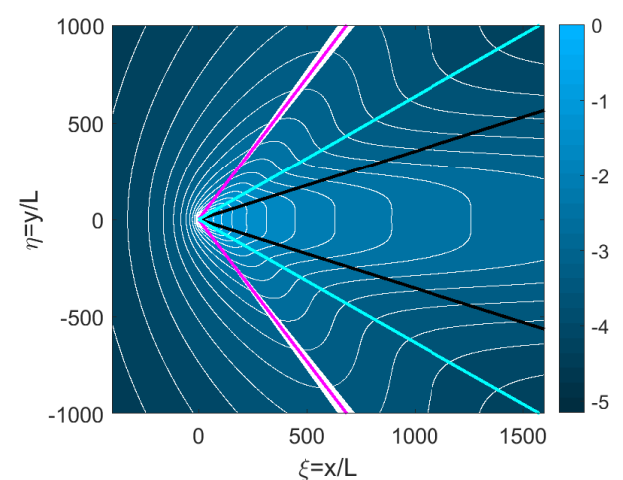

(d)

Figure 4. Cont. 


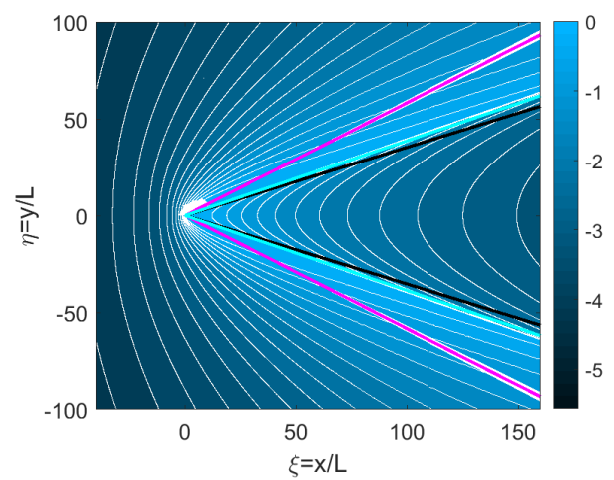

(e)

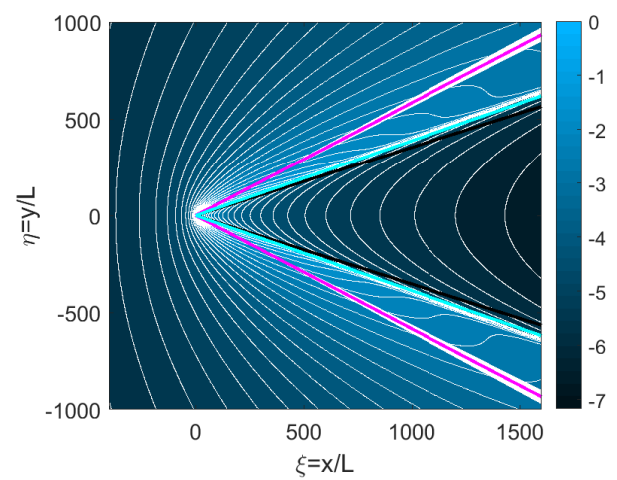

(f)

Figure 4. Isolines of wake amplitudes for the circular source in two resolutions (left and right columns). Top $-U_{*}=\sqrt{3 /(2 / \sqrt{2})}$, the first Wilton ripple resonance; middle $-U_{*}=(2 \sqrt{3}-3)^{-1 / 4}$, the minimum of the group velocity; bottom $-U_{*}=1.938$, the threshold of the wake cusp occurrence. Levels are log-spaced with decrement $2^{1 / 4}$. The demarcation line (17) is shown in magenta, the "group Mach cone" $0.7681 C_{m} / U_{*}$ - cyan, the Kelvin cone-black. The patterns qualitatively resemble experimentally observed ones by (Rousseaux and Kellay [17], cf. Figures 7 and 10).

At the low speed of the first Wilton resonance $U_{*} \approx 1.03$ (Figure $4 \mathrm{a}, \mathrm{b}$ ), the isolines of amplitudes in the wake resemble slightly distorted circles. The amplitude vanishes at the demarcation line $\kappa=1$. It decays approximately 15 times on the scale of the right panel of Figure 4 about $5 \mathrm{~m}$.

Figure $4 \mathrm{c}$, d likely reflects an effect of the group velocity minimum. The source produces a sort of bulldozing pattern with a mushroom-shaped front and a track along the axis behind. The amplitude decay is minimal near the current axis: only 6 times at a distance of about $30 \mathrm{~m}(1600 \cdot L)$ along the axis versus 30 times at oblique directions.

At higher threshold speed $U_{*}=1.983$ (Figure 4e,f), the capillary waves propagating upstream show slower decay than their gravity counterparts. The high amplitudes are concentrated near the "group Mach" cone (cyan lines). Amplitudes of the gravity waves inside this cone appear almost one order lower than in the previous case at the same distances.

\subsubsection{Wakes of Elliptic Sources}

The reference solution method, in contrast to its asymptotic counterparts, accounts for the shape of the initial perturbation. These are bandwidth parameters $\Delta_{x}, \Delta_{y}$ in formulas for wave amplitudes defined by (39) and their reciprocal values $1 / \Delta_{x}, 1 / \Delta_{y}$ that characterize the initial perturbation dimension in the coordinate space. For correct comparison, two pairs of the bandwidth parameters that satisfy an evident condition

$$
\Delta_{x} \Delta_{y}=\text { const }
$$

have been chosen, i.e., two elliptic distributions of equal square areas in the coordinate and wavenumber spaces. Figure 5 shows wave patterns for two sets of the shape parameters for two values of dimensionless speed $U_{*}$.

Case $\Delta_{x}=0.2, \Delta_{y}=0.05$ (Figure $5 \mathrm{a}, \mathrm{b}, \mathrm{e}, \mathrm{f}$ ) presents a source for which its along-current size is 4 times shorter than its front size. This source demonstrates a pronounced bulldozing effect. For $U_{*}=(2 \sqrt{3}-3)^{-1 / 4}$ (minimum of group velocity), the mushroom pattern is larger than for the circular source (cf. Figure 4c,d) and the track is essentially narrower. For higher speed $U_{*}=1.938$, a similar mushroom-shaped pattern is seen near the Mach cone (cyan lines).

Narrow source with high length-beam ratio $\Delta_{y} / \Delta_{x}=4$ shows peculiar features in Figure $5 c, d, g, h$. For $U_{*}=(2 \sqrt{3}-3)^{-1 / 4}$ (minimum of group velocity), the wake appears larger than in other cases (circle and wide sources). For $U_{*}=1.938$, the wake perturbations are concentrating near the Mach 
angle and strongly decaying both to the short capillary waves and to the interior of the Kelvin angle of gravity waves.

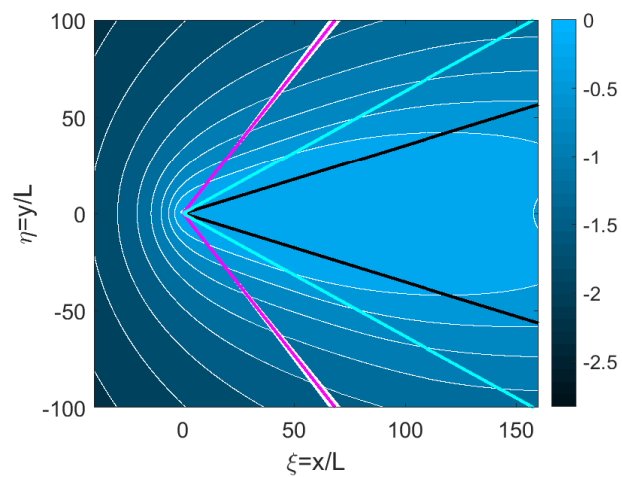

(a)

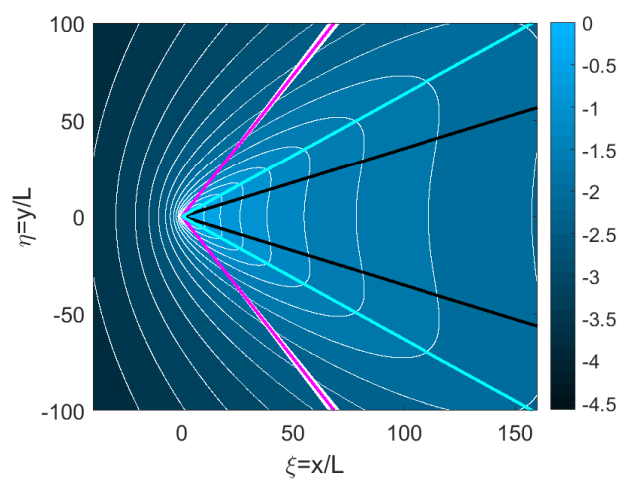

(c)

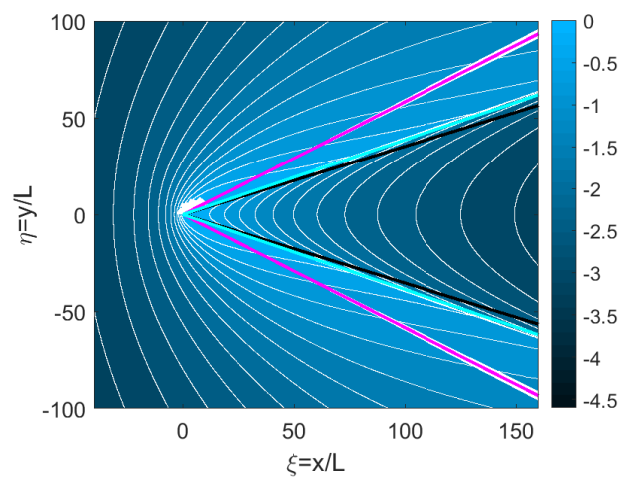

(e)

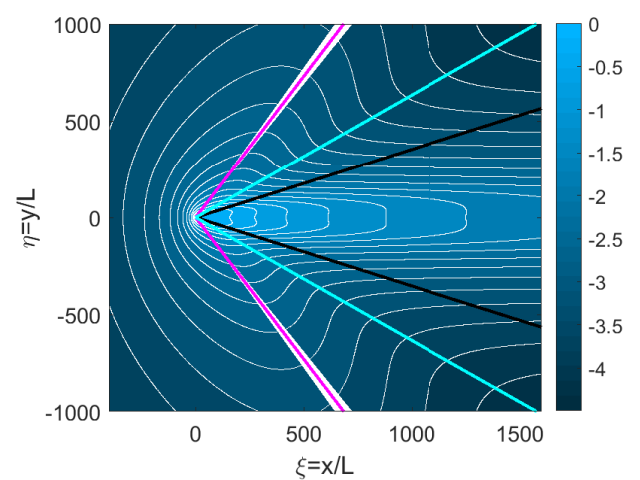

(b)

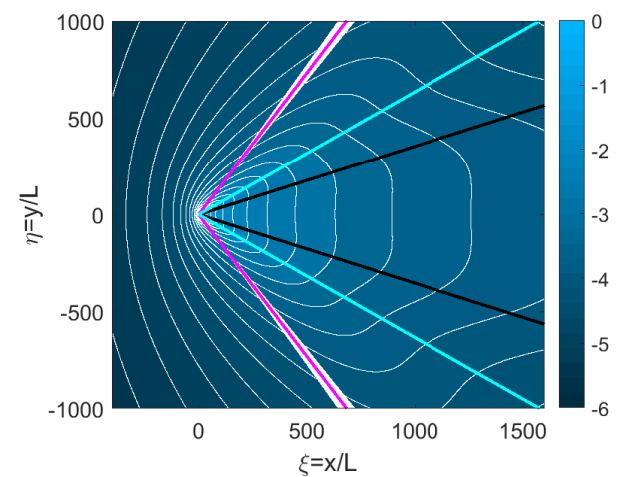

(d)

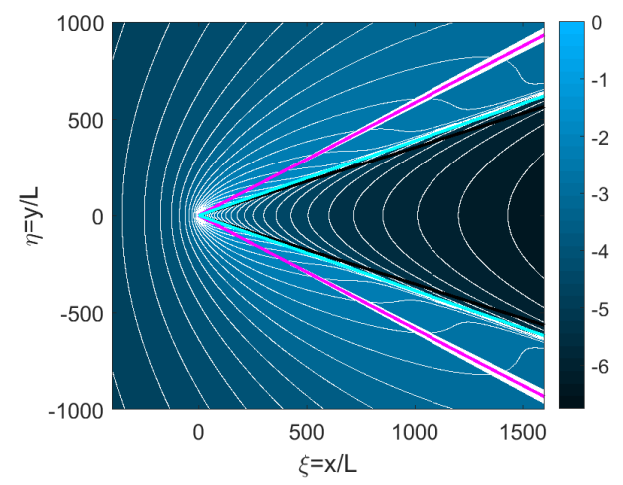

(f)

Figure 5. Cont. 


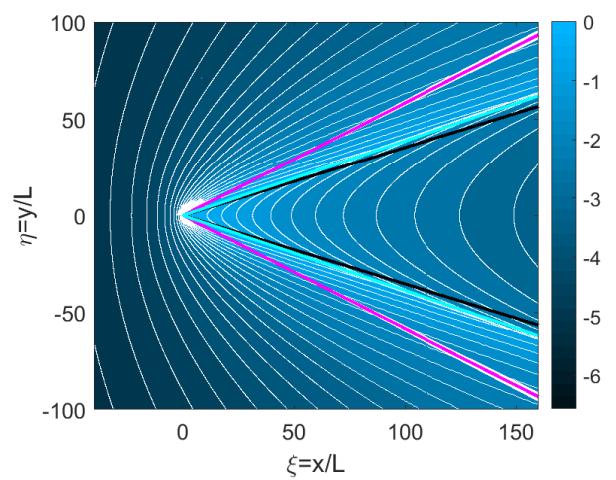

(g)

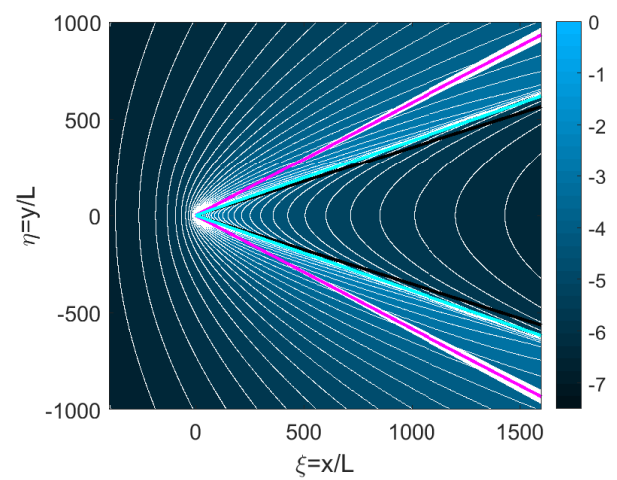

(h)

Figure 5. Isolines of wake amplitudes for the elliptic sources in two resolutions (left and right columns). $(\mathbf{a}, \mathbf{b})-U_{*}=(2 \sqrt{3}-3)^{-1 / 4}$, the minimum of the group velocity, $\Delta_{x}=0.05, \Delta_{y}=0.2 ;(\mathbf{c}, \mathbf{d})-U_{*}=$ $(2 \sqrt{3}-3)^{-1 / 4}$, the minimum of the group velocity, $\Delta_{x}=0.2, \Delta_{y}=0.0 .05 ;(\mathbf{e}, \mathbf{f})-U_{*}=1.938$, $\Delta_{x}=0.05, \Delta_{y}=0.2$ the threshold of the wake cusp occurrence; $(\mathbf{g}, \mathbf{h})-U_{*}=1.938, \Delta_{x}=0.2, \Delta_{y}=0.05$ the threshold of the wake cusp occurrence. Levels are log-spaced with decrement $2^{1 / 4}$. The demarcation line (17) is shown in magenta, the " group Mach cone" $0.7681 C_{m} / U_{*}-$ cyan, the Kelvin cone-black.

It would be interesting to verify the above estimates of the wave amplitude patterns experimentally.

\section{Discussion and Conclusions}

The gravity-capillary waves play a special role in ocean studies. One of the issues is the remote sensing applications. Satellite altimeters (Ka- and Ku-band) and other space- and airborne systems operate in the intermediate range of wavelength where the effects of gravity and capillarity compete on equal terms. This is a reason for focusing on the specific range of scales and/or relatively low dimensionless speed of $U_{*}$. In this case, wave patterns differ dramatically from the well-known pictures of pure gravity waves with the classic Kelvin cone.

We started with the analysis of the gravity-capillary wave kinematics following the conventional approach developed in previous works (e.g., [1,21-23]). The feature of our approach is in the scale choice. The wave Bond number $\kappa=|\mathbf{k}| L$ provides a useful reference $\kappa=1$ for capillary $(\kappa>1)$ and gravity $(\kappa<1)$ families of the ship wake in wavenumber and coordinate spaces. The counterpart of the Froude number, dimensionless speed of current $U_{*}=U / C_{m}$, is exploited for specifying a variety of patterns of gravity-capillary waves.

Parameters of wave patterns are specified analytically, thus extending the results of recent works (e.g., $[13,24])$. In particular, analytical expressions for parameters of cusps of isophases are presented (e.g., (22)-(24)) and related to phase and group velocity extremes, and characteristic angles (see Figure 1).

The analytical expressions for the wake amplitudes are derived with the reference solutions approach for the first time. The "phase Mach" cone is shown to be invisible because of vanishing amplitudes along the demarcation line of gravity and capillary families. On the contrary, the "group Mach" cone is emphasized by the effect of quasi-dispersion of two-dimensional waves when the corresponding dispersion factor (51) vanishes. This is in line with the analytical and experimental results of Moissy and Rabaud [13]. The amplitude effect of the "group Mach" cone is demonstrated for a limited range of dimensionless speed $U_{*} \leq 1.938$ when cusps are absent. It is natural to assume that the presence of the merging waves at the cusps enhances this effect and emphasizes the "group Mach" effect at higher speeds.

The reference solution approach goes beyond the point-wise paradigm of its asymptotic counterparts (e.g., stationary phase approach), thus accounting for the effect of the source shape. 
The corresponding estimates have been carried out for elliptic sources of different orientation. The bulldozing effect of the shapes on the wake has been demonstrated in Figure 5 fairly well.

Author Contributions: Conceptualization, formal analysis, writing-original draft preparation-V.G. Methodology, formal analysis, visualization, writing-review and editing-S.B. Both authors have read and agreed to the published version of the manuscript.

Funding: This research was funded by Russian Science Foundation Grant $\sharp 19-72-30028$ with the contribution of ITPMK Ltd, Moscow Russia

Conflicts of Interest: The authors declare no conflict of interest.

\section{References}

1. Svirkunov, P.N.; Kalashnik, M.V. Phase patterns of dispersive waves from moving localized sources. Physics-Uspekhi 2014, 57. [CrossRef]

2. Kelvin, L. Deep sea ship waves. Proc. R. Soc. Edinb. 1906, 25, 1060-1084. [CrossRef]

3. Thomson, W. XLII. On stationary waves in flowing water. Part I. Philos. Mag. Ser. 1886, 22, 353-357. [CrossRef]

4. Thomson, W. On ship waves. Proc. Inst. Mech. Eng. 1887, 38, 409-434. [CrossRef]

5. Thomson, W. Popular Lectures and Addresses, In Three Volumes; Volume III of Nature Series; Chapter On Ship Waves; McMillan and Co.: London, UK; New York, NY, USA, 1891; pp. 450-506.

6. Havelock, T.H. The propagation of groups of waves in dispersive media, with application to waves on water produced by a travelling disturbance. Proc. R. Soc. Lond. A 1908, 81, 398-430. [CrossRef]

7. Lamb, H. Hydrodynamics, 6th ed.; Dover: New York, NY, USA, 1932; 738p.

8. Lighthill, J. Waves in Fluids; Cambridge University Press: Cambridge, UK, 1978; 504p.

9. Gnevyshev, V.G.; Badulin, S.I. On reference solutions for ship waves. AIP Adv. 2020, 10, 025014. [CrossRef]

10. Rayleigh, L. The form of standing waves on the surface of running water. Proc. Lond. Math. Soc. 1883, 1, 69-78. [CrossRef]

11. Hamilton, W.R. Researches respecting vibration, connected with the theory of light. Proc. R. Irish Acad. 1841, 1, 341-349

12. Binnie, A.M. Solutions of the fish-line problem at intermediate velocities. Br. J. Appl. Phys. 1965, 16, 1755-1758. [CrossRef]

13. Moissy, F.; Rabaud, M. Mach-like capillary-gravity wakes. Phys. Rev. E 2014, 90, 023009. [CrossRef] [PubMed]

14. Gnevyshev, V.G.; Badulin, S.I. On the asymptotics of multidimensional linear wave packets: Reference solutions. Mosc. Univ. Phys. Bull. 2017, 72, 415-423 [CrossRef]

15. Liang, H.; Chen, X. Asymptotic analysis of capillary-gravity waves generated by a moving disturbance. Eur. J. Mech. B/Fluids 2018, 72, 624-630. [CrossRef]

16. Whitham, G.B. Linear and Nonlinear Waves; Wiley: New York, NY, USA, 1974; 636p.

17. Rousseaux, G.; Kellay, H. Classical hydrodynamics for analogue spacetimes: Open channel flows and thin films. Philos. Trans. R. Soc. A Math. Phys. Eng. Sci. 2020, 378, 20190233. [CrossRef] [PubMed]

18. Clauss, G.F.; Bergmann, J. Gaussian wave packets-A new approach to seakeeping tests of ocean structures. Appl. Ocean Res. 1986, 8, 190-206 [CrossRef]

19. Fedoryuk, M.V. Encyclopedia of Mathematics; Chapter Saddle Point Method; Springer: Berlin/Heidelberg, Germany; Kluwer Academic Publishers: New York, NY, USA, 1994

20. Wilton, J.R. LXXII. On ripples. Philos. Mag. Ser. 1915, 29, 688-700. [CrossRef]

21. Crapper, G.D. Surface waves generated by a travelling pressure point. Proc. R. Soc. Lond. 1964, A282, 547-558. [CrossRef]

22. Doyle, T.B.; McKenzie, J.F. Stationary wave patterns in deep water. Quaest. Math. 2013, 36, 487-500. [CrossRef]

23. Lighthill, J. Contributions to the theory of waves in nonlinear dispersive systems. J. Inst. Maths. Appl. 1965, 1, 269-306. [CrossRef] 
24. Rabaud, M.; Moisy, F. Ship wakes: Kelvin or Mach angle? Phys. Rev. Lett. 2013, 110, 214503. [CrossRef] [PubMed]

Publisher's Note: MDPI stays neutral with regard to jurisdictional claims in published maps and institutional affiliations.

(C) 2020 by the authors. Licensee MDPI, Basel, Switzerland. This article is an open access article distributed under the terms and conditions of the Creative Commons Attribution (CC BY) license (http://creativecommons.org/licenses/by/4.0/). 